\title{
Proprotein Convertase Subtilisin-Kexin Type 9 (PCSK9) Inhibitors and Cardiovascular Risk: Does a Further Analysis of the Fourier Trial Suggest Changes in the Target of Lipid Lowering Therapy?
}

\author{
Nazzareno Cervelli ${ }^{1,3} \cdot$ Giuliano Tocci $^{2,3,4}$ - Claudio Ferri ${ }^{1,3}$
}

Received: 30 November 2017/ Accepted: 16 December 2017/Published online: 5 January 2018

(C) Springer International Publishing AG, part of Springer Nature 2018

Circulating LDL-cholesterol (LDL-C) concentration is a well established, modifiable risk factor for atherosclerosis [1]. According to this, decrements in serum LDL-C levels are followed by a marked reduction in the incidence of myocardial infarction and stroke in various patient settings [2]. In this context, cardiovascular benefits have been obtained after lipid-lowering interventions in both primary and secondary prevention trials in patients with high serum LDL-C at baseline as well as in those with intermediate and even relatively low baseline serum LDL concentrations [3]. Concordantly, several meta-analyses indicate linear correlation between cardiovascular risk reduction per unit of LDL-C [2]. The European guidelines then progressively decreased the target LDL-C values to $<100 \mathrm{mg} / \mathrm{dl}$ $(2.59 \mathrm{mmol} / \mathrm{l})$ in primary prevention and $<70 \mathrm{mg} / \mathrm{dl}$ $(1.81 \mathrm{mmol} / \mathrm{l})$ in secondary prevention [4]. Following the recent The Improved Reduction of Outcomes: Vytorin Efficacy International Trial (IMPROVE-IT), in whom lowering LDL cholesterol by simvastatin + ezetimibe treatment to levels below previous targets $[53.2 \mathrm{mg}$ per deciliter $(1.4 \mathrm{mmol} / \mathrm{l})]$ provided additional benefit in

Claudio Ferri

claudio.ferri@cc.univaq.it

1 Department of MeSVA, University of L'Aquila, Coppito 67100, L'Aquila, Italy

2 Division of Cardiology, Sant'Andrea Hospital, Department of Clinical and Molecular Medicine, University of Rome Sapienza, 00189 Rome, Italy

3 The Italian Society of Hypertension (SIIA), Rome, Italy

4 IRCCS Neuromed, Pozzilli, IS, Italy patients who had been hospitalized for an acute coronary syndrome within the preceding 10 days [5], the American Association of Clinical Endocrinologists (AACE) and American College of Endocrinology (ACE) indicated the goal of $<55 \mathrm{mg} / \mathrm{dl}$ in patients at extreme cardiovascular risk [unstable angina after achieving an LDL-C $<70 \mathrm{mg} / \mathrm{dl}$, established clinical cardiovascular disease in patients with diabetes mellitus, chronic kidney disease in stages 3 or 4 , heterozygous familial hypercholesterolemia or history of premature cardiovascular disease $(<55$ male,$<65$ female) $]$ [6].

Recently, the Further cardiovascular OUtcomes Research with Proprotein Convertase Subtilisin-Kexin type 9 (PCSK9) Inhibition in subjects with Elevated Risk (FOURIER) trial showed that the inhibition of PCSK9 obtained by the monoclonal antibody evolocumab, when added to background optimal high intensity statin \pm ezetimibe therapy lowered serum LDL-C concentrations to $30 \mathrm{mg} / \mathrm{dl}(0.78 \mathrm{mmol} / \mathrm{l})$ in patients with stable vascular disease [7]. When compared to patients treated only with statin + ezetimibe, those receiving also evolocumab manifested a mean percentage reduction in serum LDL cholesterol levels of 59\% [95\% confidence interval (CI), 58-60; $P<0.001]$. The rate of cardiovascular events was significantly reduced by evolocumab versus placebo (i.e. statin with or without ezetimibe) $(-27 \%$ myocardial infarction, $-21 \%$ stroke, $-22 \%$ coronary revascularizations) during a median period of 2.2 years of follow up. Thus, in the FOURIER trial achieved LDL-C concentration were substantially lower than those observed in all of the previous clinical trials with any lipid-lowering therapy [8] including the IMPROVE-It trial [5]. 
In some preliminary analyses, the benefits obtained by the reduction in serum LDL-C levels to $30 \mathrm{mg} / \mathrm{dl}$ were claimed to be not linearly correlated to the achieved serum LDL-C concentrations [8]. Thus, the reduction in cardiovascular events seemed lower than expected and thereby suggested the existence of a plateau of the benefits obtainable by lipid lowering strategies.

In particular, a number needed to treat $(\mathrm{NNT})=74$ patients over a period of 2 years was claimed to be necessary in order to prevent 1 cardiovascular death, myocardial infarction or stroke. However, when extending the analysis to 36 months, the NNT became closer to 50 . Extrapolated to 5 years, which is the duration of the major clinical trials testing statins [9], the NNT to prevent one event is approximately $25-30$ [10]. Thus, even considering the lack of significant adverse events in patients treated with evolocumab and the evidence that $42 \%$ of evolocumab treated patients achieved LDL-C levels $<25 \mathrm{mg} / \mathrm{dl}$ without manifesting relevant side effects, including no change in cognitive function [11], benefits from the evolocumab-induced marked LDL-C reductions appeared to be not lower than expected. In keeping to this, a NNT $=29$ over 2.5 years have been recently indicated in FOURIER patient's with peripheral artery disease at baseline [12].

In conclusion, inhibition of PCSK9 with evolocumab lowered serum LDL cholesterol levels to $30 \mathrm{mg} / \mathrm{dl}$ and further reduced the risk of cardiovascular events. Although real life studies are awaited to confirm both safety and efficacy data from the FOURIER trial, lowering of LDL cholesterol levels well below the currently recommended target by PCSK9 inhibition seem to be accompanied by the expected reduction in cardiovascular events. Needless to say, awareness, treatment, and control of elevated LDL cholesterol levels are still far to achieve the expected target, particularly in low income patient subsets [13]. Thus, treatment of elevated LDL cholesterol levels in primary prevention, in patients with familial hypercholesterolemia, and in patients who already suffered from a cardiovascular event needs to be markedly improved in the real life $[14,15]$. Before prescribing evolocumab according to the Fourier protocol, clinicians should increase statin prescription and appropriate statin uptitration [16, 17]. In order to limit inappropriate prescription of biologic drugs, ezetimibe should be also more widely used in combination with statins. Concordant to this, a recent evaluation of a large cohort of veterans [18] indicated that prescription of statins, uptitration of statin therapy and addition of ezetimibe might reduce by $\approx 60 \%$ the total number of patients requiring evolocumab treatment. As a consequence, cardiovascular events would be dramatically decreased by either more appropriate statin \pm ezetimibe or evolocumab appropriate prescription, or both; with a consistent reduction in the annual cost of therapy. Thus,
PCSK9 inhibition by evolocumab seems to be accompanied by the expected reduction in cardiovascular events. A more appropriate statin \pm ezetimibe prescription, with statin uptitration, is then also a crucial step toward the more correct and cost-saving cardiovascular prevention.

\section{References}

1. Nelson RH. Hyperlipidemia as a risk factor for cardiovascular disease. Prim Care. 2013;40(1):195-211.

2. Ference BA, Ginsberg HN, Graham I, Ray KK, Packard CJ, Bruckert E, Hegele RA, Krauss RM, Raal FJ, Schunkert H, et al. Low-density lipoproteins cause atherosclerotic cardiovascular disease. 1. Evidence from genetic, epidemiologic and clinical studies. A Consensus Statement from the European Atherosclerosis Society Consensus Panel. Eur Heart J. 2017;38(32):2459-72.

3. Feher MD. Lipid lowering to delay the progression of coronary artery disease. Heart. 2000;89:451-8.

4. Catapano AL, Graham I, De Backer G, et al. 2016 ESC/EAS guidelines for the management of dyslipidaemias. Eur Heart $\mathrm{J}$. 2016;37:2999-3058.

5. Cannon CP, Blazing MA, Giugliano RP. IMPROVE-IT investigators. Ezetimibe added to statin therapy after acute coronary syndromes. N Engl J Med. 2015;18(372):2387-97.

6. Jellinger PS, Handelsman Y, Rosenblit PD, et al. American association of clinical endocrinologists and American College of endocrinology guidelines for management of dyslipidemia and prevention of cardiovascular disease. Endocr Pract. 2017;23:1-87.

7. Sabatine MS, Giugliano RP, Keech AC, Honarpour N, Wiviott SD, Murphy SA, et al. Evolocumab and clinical outcomes in patients with cardiovascular disease. $\mathrm{N}$ Engl J Med. 2017;376:1713-22.

8. Giugliano RP, Pedersen TR, Park JG, De Ferrari GM, Gaciong ZA, Ceska R, Toth K, Gouni-Berthold I, Lopez-Miranda J, Schiele F, Mach F, Ott BR, Kanevsky E, Pineda AL, Somaratne R, Wasserman SM, Keech AC, Sever PS, Sabatine MS, FOURIER Investigators. Clinical efficacy and safety of achieving very low LDL-cholesterol concentrations with the PCSK9 inhibitor evolocumab: a prespecified secondary analysis of the FOURIER trial. Lancet. 2017;390(10106):1962-71.

9. Sacks FM, Pfeffer MA, Moye L, et al. Rationale and design of a secondary prevention trial of lowering normal plasma cholesterol levels after acute myocardial infarction: the Cholesterol and Recurrent Events trial (CARE). Am J Cardiol. 1991;68:1436-46 (Erratum, J Cardiol 1992;69:574).

10. Sabatine MS. Proprotein convertase subtilisin/kexin type 9 (PCSK9) inhibitors: the next chapter in cardiovascular disease prevention. European Society of Cardiology (ESC) Congress 2017.

11. Stamerra CA, Di Giosia P, Giorgini P, De Feo M, Grassi D, Ferri C, Sahebkar A. Neurocognitive performance after PCSK9 inhibitor therapy: current state of the evidence. J Neurosci Res. 2017. https://doi.org/10.1002/jnr.24199.

12. Bonaca MP, Nault P, Giugliano RP, Keech AC, Pineda AL, Kanevsky E, Kuder J, Murphy SA, Jukema JW, Lewis BS, Tokgozoglu L, Somaratne R, Sever PS, Pedersen TR, Sabatine MS. Low-density lipoprotein cholesterol lowering with evolocumab and outcomes in patients with peripheral artery disease: insights from the FOURIER Trial (Further cardiovascular outcomes research with PCSK9 inhibition in subjects with elevated risk). 
Circulation. 2017. https://doi.org/10.1161/CIRCULATIONAHA. 117.032235 .

13. Egan BM, Li J, Sarasua SM, Davis RA, Fiscella KA, Tobin JN, Jones DW, Sinopoli A. Cholesterol control among uninsured adults did not improve from 2001-2004 to 2009-2012 as disparities with both publicly and privately insured adults doubled. J Am Heart Assoc. 2017;6(11):e006105.

14. Agabiti Rosei E, Salvetti M. Management of hypercholesterolemia, appropriateness of therapeutic approaches and new drugs in patients with high cardiovascular risk. High Blood Press Cardiovasc Prev. 2016;23(3):217-30.

15. Volpe M, Volpe R, Gallo G, Presta V, Tocci G, Folco E, Peracino A, Tremoli E, Trimarco B, Italian Society of Cardiovascular Prevention (SIPREC) Writing Committee. 2017 Position paper of the italian society for cardiovascular prevention (SIPREC) for an updated clinical management of hypercholesterolemia and cardiovascular risk: executive document. High Blood Press Cardiovasc Prev. 2017;24(3):313-29.

16. Colletti A, Derosa G, Cicero AF. Retargeting the management of hypercholesterolemia-focus on evolocumab. Ther Clin Risk Man. 2016;12:1365-76.

17. Pucci G, Cicero AF, Borghi C, Schillaci G. Emerging biologic therapies for hypercholesterolaemia. Expert Opin Biol Ther. 2017;17(9):1077-87.

18. Virani SS, Akeroyd JM, Nambi V, Heidenreich PA, Morris PB, Nasir K, Michos ED, Bittner VA, Petersen LA, Ballantyne CM. Estimation of eligibility for proprotein convertase subtilisin/kexin type 9 inhibitors and associated costs based on the FOURIER trial (further cardiovascular outcomes research with PCSK9 inhibition in subjects with elevated risk): insights From the Department of Veterans Affairs. Circulation. 2017;135(25):2572-4. 